\title{
Forest Vegetation of Hardwood Tree Species along the Mirna River in Istria (Croatia)
}

\author{
Joso Vukelić*1, Patrik Korijan ${ }^{1} \dagger$, Irena Šapić ${ }^{1}$, Antun Alegro², Vedran Šegota², Igor Poljak ${ }^{3}$
}

(1) University of Zagreb, Faculty of Forestry, Department of Forest Ecology and Silviculture, Svetošimunska 25, HR-10000 Zagreb, Croatia; (2) University of Zagreb, Faculty of Science, Department of Biology, Rooseveltov trg 6, HR-10000 Zagreb, Croatia; (3) University of Zagreb, Faculty of Forestry, Department of Forest Genetics, Dendrology and Botany, Svetošimunska 25, HR-10000 Zagreb, Croatia; (†) Deceased

* Correspondence: e-mail: jvukelic@sumfak.hr
Citation: VUKELIĆ J, KORIJAN P, ŠAPIĆ I, ALEGRO A, ŠEGOTA V, POLJAK I 2018 Forest Vegetation of Hardwood Tree Species along the Mirna River in Istria (Croatia). Southeast Eur for 9 (1): 1-16. DOI: https://doi. org/10.15177/seefor.18-05

Received: 22 Feb 2018; Revised: 5 May 2018; Accepted: 8 May 2018; Published online: 5 Jun 2018

\begin{abstract}
Background and Purpose: The paper presents the forest vegetation of periodically flooded and wet forests of hardwood tree species along the Mirna River (Istria, Croatia). The main objective was to study the older and less influential stands, and to compare them among themselves and with related syntaxa of the Mediterranean and continental area.

Materials and Methods: The research was conducted on the basis of 33 new phytocoenological relevés and 12 from previous studies, according to the principles of the standard Central European Phytocoenological School.

Results and Conclusions: Based on 45 phytocoenological relevés two main vegetation types were found. In the lowest and periodically flooded habitats grow forests dominated by Fraxinus angustifolia and numerous hygrophilous species. In somewhat higher and drier localities, but with a high level of ground waters, grow mixed forests of Quercus robur, Fraxinus angustifolia, Ulmus minor and Carpinus betulus, with a greater presence of mesophilous species. The paper analyzes their mutual relationship, phytocoenological affiliation, as well as their position with regard to the related syntaxa of the Mediterranean and continental area. The results suggest isolation and a transitional character of the studied forests, which is a consequence of the biogeographical position in the north Mediterranean, of the ecological conditions, and to a lesser extent of anthropogenic influence.
\end{abstract}

Keywords: hardwood forests, Mirna River (Istria), floristic composition, differential species, flooded and wet habitats

\section{INTRODUCTION}

Mediterranean forests of hardwood trees are very rare nowadays, and one of the best-preserved ones is located on the Istrian Peninsula (Croatia) in the lower course of the Mirna River (better known as the Motovun Forest). The forests along the course of the Mirna River stretch over approximately 1100 ha, and the main tree species are Quercus robur, Fraxinus angustifolia and Ulmus minor, whereas the drier part also holds Acer campestre and Carpinus betulus. The basic ecological factor for the composition and growth of these forests is the periodically flooding and high groundwater, which reflects on the floristic composition and distribution of syntaxa. In addition, the biogeographical position is specific as it is in the north of the Mediterranean and close to the Dinaric massif, with a strong continental influence.
The first phytocoenological studies [1, 2] presented the entire complex of the Motovun Forest as an autonomous association Querco robori-Carpinetum betuli "submediterraneum" within the southeast European alliance of oak-hornbeam forests Erythronio-Carpinion betuli. This was subsequently, following corrections to the name of the association, also accepted by other phytocoenologists $[3,4]$. Brullo and Spampinato [5] classify them in the association Querco roboris-Carpinetum betuli within the alliance Alno-Quercion roboris. Finally, Trinajstic [6] defined them descriptively as a new association Carici pendulae-Ouercetum roboris, also within the alliance Alno-Quercion roboris. Vukelić [7] advocates for more intensive pyhtocoenological research because the 2009 forest inventory suggests differences in the 
composition and structure of stands. According to Vukelić [7], it is not possible to cover the entire forest complex with a single syntaxa.

These different views on nomenclature and syntaxonomy have impelled us to conduct phytocoenological studies of these forest stands in 2016 and 2017. The objective was to survey older and less influential stands, and to compare them among themselves and with related syntaxa of the Mediterranean and continental area. The results of the research should help to define the forest vegetation of this important forest locality, but also of the northern part of the Mediterranean region. When it comes to these issues, the opinions of phytocoenologists are quite varied, which is also evidenced by the overviews of the forest vegetation of Europe or its specific regions [5, 8-12].

\section{MATERIAL AND METHODS}

\section{Research Area}

The Motovun Forest is situated in the western part of the Istrian Peninsula, in the valley of the Mirna River (43 km long) and its Butoniga tributary (Figure 1). It is some fifteen kilometers away from the sea coast, and only a few more kilometers away from the Dinaric massif Ćićarija (peak Orljak, $1106 \mathrm{~m}$ ). The terrain altitude ranges between 7 and $17 \mathrm{~m}$, and the forest complexes stretch along $15 \mathrm{~km}$, with the average width of approximately $500 \mathrm{~m}$. Nowadays, the Motovun Forest is known Europe-wide as the habitat of the white and black truffle (Tuber magnatum and Tuber melanosporum), and the habitat of the Italian agile frog (Rana latastei). It is a part of the Natura 2000 ecological network, and 300 ha of the old forest were protected in 1963 as a "special reserve of forest vegetation".

This area is characterized by a moderately warm and humid climate with hot summers. According to the data from the Botoniga weather station (29 m MASL, period 19862015 ) the mean annual temperature is $13.0^{\circ} \mathrm{C}$, and the mean annual precipitation is $1004 \mathrm{~mm}$. For comparison, the mean annual temperature in the area of Abruzzo and Molise in the central part of the Adriatic coast in Italy is higher by up to $4^{\circ} \mathrm{C}$, and precipitation is lower by up to $400 \mathrm{~mm}$ [13] than in similar conditions in Istria.

Parent material of the Motovun Forest is composed of Eocene marls and sandstone washed down by torrents from the surrounding hills and deposited into the valley of the Mirna and Butoniga rivers. Periodic floods, ground waters and constant depositing of new detritus cause the creation of different hydromorphic soils. They are characterized by excessive wetting and are in the development stage of pseudogley and gley-pseudogley.

The valley of the Mirna River is surrounded by the zonal vegetation of the downy oak and oriental hornbeam (Querco pubescenti-Carpinetum orientalis), with a more pronounced featuring of continental beech and oak-beech forests. Thus in the forests of the Mediterranean region we find species of the Illyrian floristic geo-element such as Primula vulgaris, Lonicera caprifolium, Knautia drymeia subsp. drymeia and others species characteristic of the order Fagetalia and lower syntaxa.

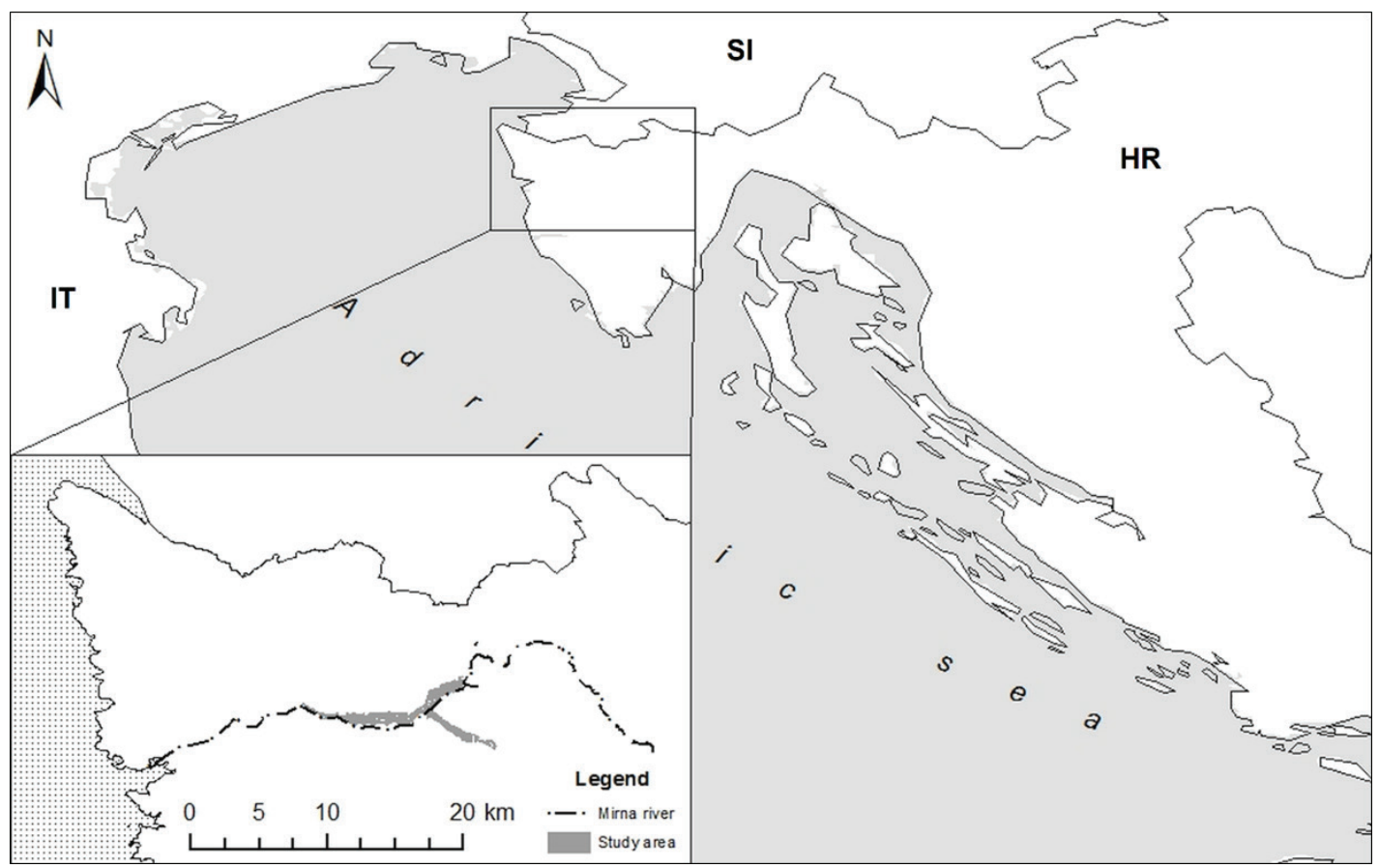

FIGURE 1. Geographical position of the study area. 


\section{Data Sampling}

Studies of the forest vegetation were conducted following the principles of the standard phytocoenological school [14]. The field phytocoenological survey was conducted on 28 plots in the period from April to mid-July 2016, whereas five unpublished relevés of Fraxinus angustifolia stands originate from the ecological vegetation study of hydrological issues of the Motovun Forest [15]. In addition to these relevés, the statistical analysis also includes 12 relevés from previous studies [2]. Positions of new relevés in the WGS84 Coordinate Reference System are listed in the Appendix 1. The surface of the plots was $400 \mathrm{~m}^{2}$, inclination $0^{\circ}$, and the terrain altitude ranged between 10 and $18 \mathrm{~m}$. The plant nomenclature is aligned with the Flora Croatica Database [16], and mosses with Atherton et al. [17]. Syntaxonomic nomenclature of higher units mostly follows the overviews by Biondi et al. [18] for Fraxinus angustifolia stands, and Košir et al. [19] for mixed hardwood stands. The local syntaxa from previous studies are presented in their original form, whereby a part of the syntaxa is described according to ICPN [20], and a part of them followed the multidimensional distribution of vegetation units [21]. The original names of the syntaxa with their authors and year of publication are listed in the supplementary materials (Appendix 2).

\section{Data Analysis}

Vegetation relevés were entered into the TURBOVEG database [22]. Cover-abundance values of the species appearing in several layers were combined in the TURBOVEG program [22], with every plant species being considered with total cover-abundance, regardless of the number of structural layers of the individual relevé in which it appears. Hierarchical clustering was performed in the $\mathrm{R}$ package (www.r-project.org) [23]. Bray-Curtis dissimilarity index [24], and square-root transformation of species percentage coverabundance values were used. In the phytocoenological analysis, we separated our 33 analytical relevés into two types and compared them with the presence class of related phytocoenoses of the Mediterranean and continental part of the southwestern rim of the Pannonian Plain. The diagnostic species were determined using the JUICE 7.0 program [25], based on the analysis of fidelity measure.

The mosses were not recorded in the majority of studies, hence they were not taken into consideration in statistical analyses. Individual species and subspecies were consolidated under a species aggregate.

\section{RESULTS AND DISCUSSION}

The statistical analysis clearly classifies the 33 relevés of the Motovun Forest into two clusters or vegetation types. The first type with 23 phytocoenological relevés represents mixed stands of Quercus robur, Fraxinus angustifolia, Ulmus minor and Carpinus betulus, whereas the second type with 10 phytocoenological relevés is dominated by Fraxinus angustifolia. Both types were in further analyses separately compared to the floristically and chorologically related syntaxa of southern Europe and continental forests of the southwestern part of the Pannonian Plain - western part of the Pannonian sector of the Pannonian-Carpathian floristic province sensu [26]. All of the compared syntaxa are listed in Table 1 under their original names, authors, and numbers of relevés.

\section{Forests Dominated by Fraxinus angustifolia}

The dendrogram on Figure 2 compares the presence classes of 16 Fraxinus angustifolia syntaxa in 23 columns. Of the syntaxa of the Mediterranean area, the most significant association is Carici remotae-Fraxinetum oxycarpae, while continental forests are represented by the association Leucojo-Fraxinetum angustifoliae. The dendrogram clearly separates Mediterranean forests of narrow-leaved ash from continental forests. In the first cluster which encompasses Mediterranean forests of narrow-leaved ash, two subclusters are clearly singled out. The studied forests along the Mirna River are classified in the sub-cluster 1a representing the association Carici remotae-Fraxinetum oxycarpae. Accordingly, we attached them to this association.

The association Carici remotae-Fraxinetum oxycarpae was established in the territory of Abruzzo in Italy [27, 28], and later on also in other parts of the Apennine Peninsula [29-35] and southern Europe [36, 37]. This community occupies lowland localities along river banks or depressions between deposits which are under the influence of the periodically flooding or high groundwater. According to the majority of cited papers, the diagnostically most important species for this association are: Fraxinus angustifolia, Carex remota, Ulmus minor, Rumex sanguineus, Ranunculus lanuginosus, Oenanthe pimpinelloides, Carex pendula and Carex divulsa.

In the studied forests along the Mirna River, the dominant ash stands stretch over several separate localities in the wettest and lowest parts of the studied area. These are shallower depressions with occasional surface waters. The tree layer is characterized by the complete domination of the narrow-leaved ash and co-domination of the European white elm, while the common oak is individually rarely present, and the common hornbeam is completely missing. In addition to the ash, elm and the mentioned diagnostic species, in the composition of the studied forests sociologically important are the species common in flooded and wet forests (such as Carex riparia, Lycopus europaeus, Cardamine pratensis, Leucojum aestivum, Galium palustre, Ranunculus repens, Lysimachia nummularia and others).

In its great area, the association Carici remotaeFraxinetum oxycarpae demonstrates floristic variability, which was also demonstrated in the stands we studied in Croatia. They hold a number of species that have not been reported or are rare in other regions. These are primarily Crataegus laevigata, Cardamine pratensis, Deschampsia cespitosa, Lysimachia nummularia, Alisma plantago-aquatica and Lycopus europaeus. These species were singled out as differential for the new sub-association crataegetosum laevigatae, the holotype of which is relevé number 4 in Table 2 (according to Weber et al. [20]). Great diagnostic significance is also proper to Carex riparia, although it has already been set as a differential species of the sub-association Carici remotae-Fraxinetum oxycarpae caricetosum cuprinae [37].

Besides the association Carici remotae-Fraxinetum oxycarpae, in southern Europe several similar syntaxa have 


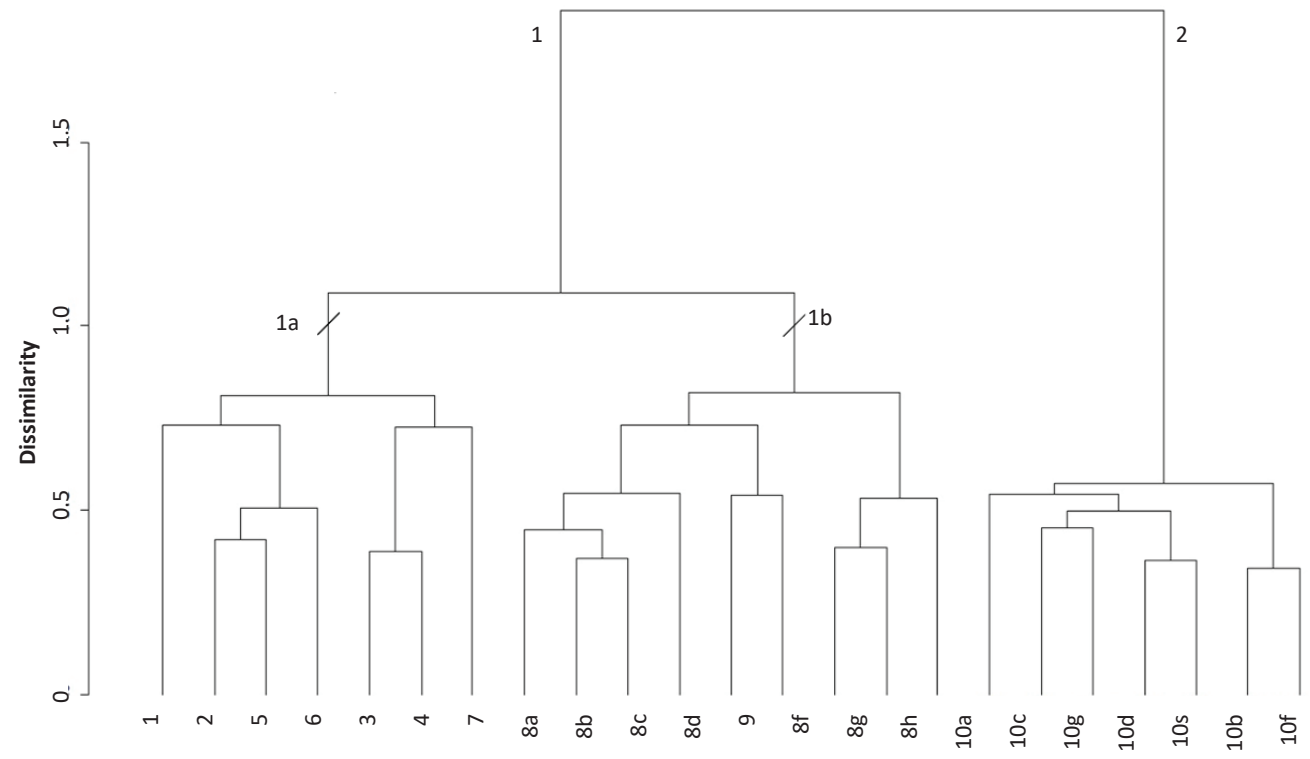

FIGURE 2. Dendrogram of the compared Fraxinus angustiflolia syntaxa.

been described with the domination of Fraxinus angustifolia [5, 38-43]. Douda et al. [10] consider the majority of them to be synonyms for the association Lithospermo purpureocaerulei-Ulmetum minoris, which they included into the middle Mediterranean alliance Populion albae. The studied forests in Istria fit only partially into such a broadly understood association and its affiliation to the alliance Populion albae. They lack important diagnostic species for this association and alliance such as Bryonia dioica, Rubus ulmifolius, Iris foetidissima, Asparagus acutifolius, Celtis australis, Rubia peregrina, Smilax aspera, Oenanthe pimpinelloides, Rosa sempervirens, Tamus communis, Populus alba and others.

In the previous studies, the community Carici remotaeFraxinetum oxycarpae was classified into different alliances: Alno-Ulmion, Alno-Quercion roboris, Alnion incanae, Carici remotae-Fraxinion oxycarpae, Populion albae $[5,9,10,18$, $29,31,37]$. Our research shows a high coincidence with the alliance Carici remotae-Fraxinion oxycarpae, as it is defined in the syntaxonomic overview of the vegetation of Italy [9, 18]. In the cited papers, the species Fraxinus angustifolia subsp. oxycarpa, Ulmus minor subsp. minor, Ranunculus lanuginosus, Carex remota, Rumex sanguineus, Carex pendula and $C$. divulsa are listed as diagnostic species for it. Without going into the internal differentiation of the species Fraxinus angustifolia and Ulmus minor, the composition of species corresponds very well to the studied stands along the Mirna River. In the latest review of the vegetation of
Europe [12], the Carici remotae-Fraxinion oxycarpae is listed as a synonym for the alliance Lauro nobilis-Fraxinion angustifoliae. However, the issue of syntaxonomy will continue to be the subject of many discussions.

Mixed Forests Dominated by Quercus robur, Fraxinus angustifolia, Ulmus minor and Carpinus betulus

Mixed hardwood forests of the studied area are presented with 23 new relevés in Table 3. They grow in drier terrains which are up to $2 \mathrm{~m}$ higher than the previous community. They are flooded only in some areas and very rarely, only in extremely wet years in early spring or late fall. The type of soil is pseudogley on alluvial deposits, and such conditions have also reflected on the floristic composition with fewer hygrophytes and more mesophilous species.

The first phytocoenological relevés of such stands were published by Lausi [44] from northeastern Italy along the Po River, and somewhat later by Bertovic [2] from the valley of the Mirna River in the Croatian part of Istria. They described them as autonomous associations under different but invalid names, hence Marinček [45] suggested the name Asparago tenuifolii-Quercetum roboris. The majority of papers classify the studied forests into the alliance Erythronio-Carpinion [1-4, 19]. However, Brullo \& Spampinato [5] and Trinajstić [6] classify them into the alliance Alno-Quercion roboris. Due to the different nomenclature and syntaxonomic characterization and their status, we surveyed them phytocoenologically in more detail and in further analyses 
compared them with 10 related syntaxa in 23 synthetic columns (Table 1, Figure 3). The compared syntaxa originate from the north-Adriatic Mediterranean area and southwestern rim of the Pannonian Plain.

The statistical analysis demonstrated the separation of the compared syntaxa into two main clusters: the first one represents drier communities with a significant presence of mesophilous species from alliances within the order Fagetalia, and the other one contains communities in periodically flooded and moist habitats with the main subassociation Genisto elatae-Quercetum roboris caricetosum remotae (Table 1 numbers 15 a-b, 16 a-f, and 19) within the alliance Alnion incanae. In the first cluster, one can distinguish between three sub-clusters: the first one represents Carpinetum communities of central and eastern Croatia and the studied area (1a); the second one more humid syntaxa of Slovenia classified in the alliances Alnion incanae and Fraxino angustifoliae-Carpinion betuli (1b); and the third one the association Fraxino pannonicaeQuercetum roboris from northern Italy, which is classified in the alliance Alno-Quercion roboris (1c).

The studied forests in the valley of the Mirna River demonstrate affiliation with the sub-cluster $1 a$, where the dominant forests are oak-hornbeam forests of drier type of central and eastern Croatia. They grow along the course of the Sava River and are connected to the studied forests in Istria, primarily by the great presence of Carpinus betulus, Acer campestre and other mesophilous species. The floristic and sociological relationships between individual cluster groups were analyzed based on the fidelity of species using the JUICE 7.0 program [25]. We took a total of 216 phytocoenological relevés in the analysis from the main syntaxa representing an individual group (Table 4).
The analysis has shown that the studied forests of the Mediterranean area differ from continental forests in wetter habitats (cluster 2 - Genisto elatae-Quercetum roboris caricetosum remotae = Fraxino pannonicaeUlmetum glabrae sensu Douda et al. 2016.) in the lack of a large number of species of wetland and flooded habitats (for instance, Iris pseudacorus, Genista tinctoria, Stachys palustris, Caltha palustris, Carex strigosa, Mentha aquatica, Carex vesicaria, C. elongata, Lythrum salicaria, Euphorbia palustris, Myosotis palustris etc.). In relation to the Mediterranean hardwood forests (subcluster 1c, column 4 in Table 1) in forests along the Mirna River, there are no southern European species represented in the communities of the alliance Populion albae. These species are Iris foetidissima, Rubus ulmifolius, Quercus ilex, Holcus lanatus, Populus alba, Cyclamen repandum, Hypericum androsaemum, Moehringia trinervia, Luzula forstery, and with a smaller presence in the Mediterranean forests there are also Aristolochia pallida, Laurus nobilis, Smilax aspera, Rosa sempervires, Clematis vitalba, Asparagus acutifolius and other species.

The studied stands demonstrate a differential character towards all of the compared syntaxa through a higher presence of the species Primula vulgaris, Vinca minor, Lonicera caprifolium, Ranunculus lanuginosus, Arum italicum, Carex pendula, Ligustrum vulgare, Corylus avellana, Ruscus aculeatus, Viburnum opulus, Aegopodium podagraria, Listera ovata, Cornus sanguinea, Symphytum tuberosum, and also Carpinus betulus, Acer campestre and Ulmus minor. The majority of these species tend to be distributed in the drier oak-hornbeam and beech forests (alliances Carpinion betuli, Erythronio-Carpinion, order Fagetalia). Hence, in the majority of studies to date, they

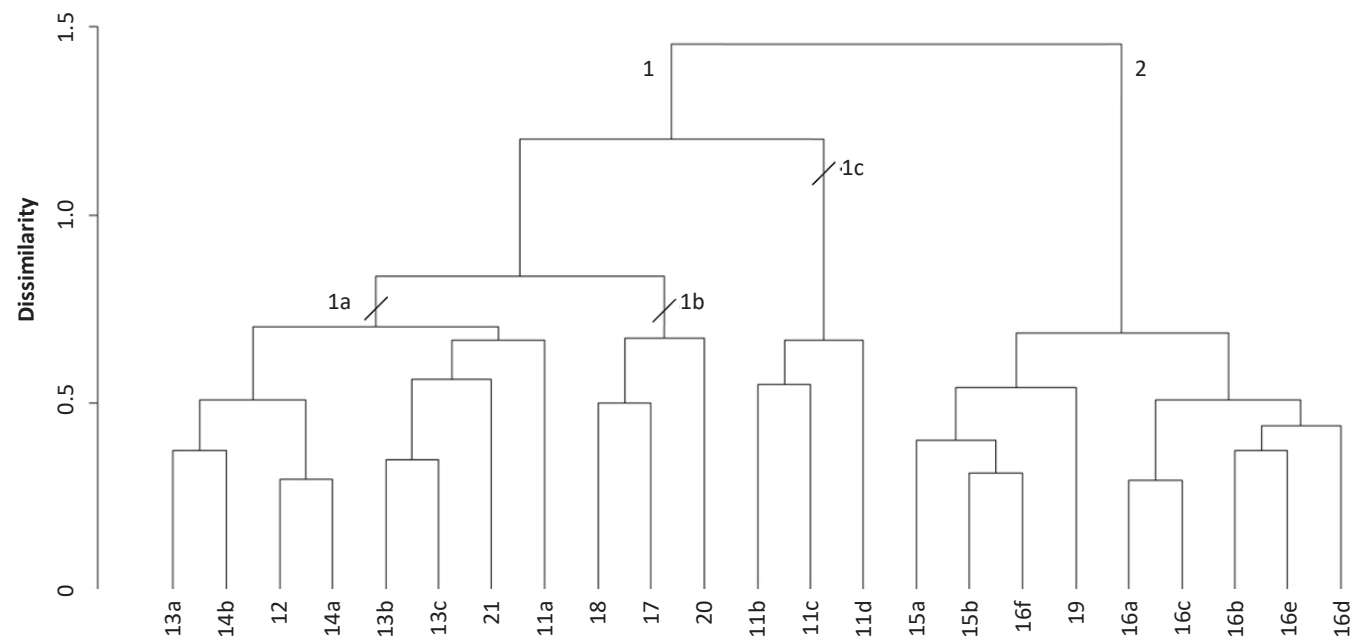

FIGURE 3. Dendrogram of the compared mixed hardwood syntaxa. 
TABLE 1. List of literature sources and phytocoenological relevés in the analysis. The numbers of relevés correspond to the numbers in the dendrogram on Figure 2 and Figure 3.

\begin{tabular}{|c|c|c|}
\hline Syntaxa & Source & No of relevés \\
\hline \multicolumn{3}{|l|}{ Fraxinus angustifolia forests (Figure 2) } \\
\hline \multicolumn{3}{|l|}{ Mediterranean area } \\
\hline 1. Corno-Fraxinetum angustifoliae & Mercedal \& Vilar [37] & 6 \\
\hline 2. Viburno lantanae-Ulmetum minoris & Mercedal \& Vilar [37] & 39 \\
\hline 3. Ficario ranunculoidis-Fraxinetum angustifoliae & Mercedal \& Vilar [37] & 77 \\
\hline 5. Rusco-Fraxinetum angustifoliae & Gesti et al. [42] & 34 \\
\hline 6. Alno glutinosae-Fraxinetum angustifoliae & Mercedal \& Vilar [37] & 23 \\
\hline 7. Junco-Fraxinetum oxycarpae & Mercedal \& Vilar [37] & 4 \\
\hline 8a. Carici remotae-Fraxinetum oxycarpae populetosum albae & Pedrotti [27] & 5 \\
\hline 8b. Carici remotae-Fraxinetum oxycarpae populetosum albae & Pedrotti \& Cortini Pedrotti [29] & 17 \\
\hline 8c. Carici remotae-Fraxinetum oxycarpae populetosum albae & Pedrotti [30] & 8 \\
\hline 8d. Carici remotae-Fraxinetum oxycarpae alnetosum glutinosae & Gellini et al. [31] & 8 \\
\hline 8e. Carici remotae-Fraxinetum oxycarpae iridetosum foetidissimae & Conti \& Pirone [33] & 10 \\
\hline 8f. Carici remotae-Fraxinetum oxycarpae quercetosum roboris & Mercedal \& Vilar [37] & 12 \\
\hline 8g. Carici remotae-Fraxinetum oxycarpae quercetosum pubescentis & Mercedal \& Vilar [37] & 9 \\
\hline 8h. Carici remotae-Fraxinetum oxycarpae caricetosum cuprinae & Mercedal \& Vilar [37] & 11 \\
\hline 9. Carici remotae-Fraxinetum oxycarpae crataegetosum laevigatae & this paper & 10 \\
\hline \multicolumn{3}{|l|}{ Continental area } \\
\hline 10a. Leucojo-Fraxinetum typicum and alnetosum glutinosae & Glavač [49] & 23 \\
\hline 10b. Leucojo-Fraxinetum typicum & Baričević [50] & 5 \\
\hline 10c. Leucojo-Fraxinetum typicum & Škvorc et al. [51] & 14 \\
\hline 10d. Leucojo-Fraxinetum alnetosum glutinosae & Rauš [46] & 5 \\
\hline 10e. Leucojo-Fraxinetum alnetosum glutinosae & Rauš [52] & 5 \\
\hline
\end{tabular}

$\frac{\text { 10g. Leucojo-Fraxinetum alnetosum glutinosae }}{\text { Mixed hardwood forests (Figure 3) }}$

Mediterranean area

\begin{tabular}{|c|c|c|}
\hline 11a. Fraxino-Quercetum roboris & Gellini et al. [31] & 15 \\
\hline 11b. Fraxino-Quercetum roboris & Manzi [32] & 5 \\
\hline 11c. Fraxino-Quercetum roboris & Brullo \& Spampinato [35] & 15 \\
\hline \multicolumn{3}{|l|}{ Continental area } \\
\hline 12. Querco roboris-Carpinetum & Glavač [54] & 33 \\
\hline 13b. Carpino betuli-Quercetum roboris typicum & Rauš [47] & 15 \\
\hline 13c. Carpino betuli-Quercetum roboris typicum & Škvorc et al. [51] & 21 \\
\hline 14a. Genisto-Quercetum roboris carpinetosum betuli & Glavač [56] & 8 \\
\hline 14b. Genisto-Quercetum roboris carpinetosum betuli & Baričević [50] & 10 \\
\hline 15a. Genisto-Quercetum roboris caricetosum brizoidis & Rauš [52] & 10 \\
\hline 15b. Genisto-Quercetum roboris caricetosum brizoidis & Baričević [50] & 10 \\
\hline 16b. Genisto-Quercetum roboris caricetosum remotae & Rauš [46] & 5 \\
\hline 16c. Genisto-Quercetum roboris caricetosum remotae & Rauš [47] & 10 \\
\hline 16d. Genisto-Quercetum roboris caricetosum remotae & Cestar et al. [55] & 31 \\
\hline 16e. Genisto-Quercetum roboris caricetosum remotae & Glavač [58] & 34 \\
\hline 16f. Genisto-Quercetum roboris caricetosum remotae & Baričević [50] & 10 \\
\hline 17. Pseudostellario europaeae-Carpinetum betuli & Dakskobler [59] & 14 \\
\hline 18. Pseudostellario-Carpinetum betuli leucojetosum aestivi & Dakskobler [59] & 9 \\
\hline 19. Pseudostellario-Quercetum roboris leucojetosum aestivi & Dakskobler [59] & 11 \\
\hline 20. Fraxino-Ulmetum effusae quercetosum roboris & Košir et al. [60] & 20 \\
\hline 21. Asparago tenuifolii-Quercetum roboris & Bertović [2] + this paper & 35 \\
\hline
\end{tabular}


TABLE 2. Forests dominated by Fraxinus angustifolia.

\begin{tabular}{|c|c|c|c|c|c|c|c|c|c|c|c|c|}
\hline Number of releve & & 1 & 2 & 3 & 4 & 5 & 6 & 7 & 8 & 9 & 10 & \\
\hline Number in Figure 1 & & 24 & 25 & 27 & 28 & 26 & 29 & 32 & 30 & 33 & 31 & \\
\hline Altitude in $\mathrm{m}$ & & 14 & 14 & 14 & 14 & 14 & 10 & 14 & 11 & 12 & 12 & \\
\hline Releve area $\left(00 \mathrm{~m}^{2}\right)$ & & 4 & 4 & 4 & 4 & 4 & 5 & 5 & 5 & 5 & 5 & Pres. \\
\hline Cover in \%: tree layer (a) & & 80 & 80 & 85 & 80 & 80 & 60 & 80 & 60 & 70 & 65 & deg. \\
\hline schrub layer (b) & & 80 & 90 & 40 & 40 & 70 & 50 & 70 & 70 & 40 & 80 & \\
\hline herb layer (c) & & 90 & 70 & 100 & 95 & 100 & 90 & 70 & 100 & 80 & 95 & \\
\hline moos layer (d) & & 1 & 15 & 5 & 1 & 1 & - & - & - & - & - & \\
\hline \multicolumn{13}{|c|}{ Diagnostic species of the association and alliance } \\
\hline Fraxinus angustifolia* & a & 5 & 5 & 5 & 5 & 5 & 3 & 3 & 3 & 4 & 3 & 5 \\
\hline Fraxinus angustifolia & $b$ & + & 1 & 1 & 1 & 1 & 2 & + & 2 & 2 & 3 & 5 \\
\hline Carex remota* & c & 1 & 3 & 3 & 1 & 2 & 3 & + & 2 & 2 & 2 & 5 \\
\hline Rumex sanguineus* & & + & 1 & + & + & + & 2 & & 2 & + & 2 & 5 \\
\hline Fraxinus angustifolia & & + & + & & + & & + & + & 2 & + & + & 4 \\
\hline Ranunculus lanuginosus* & & 2 & + & + & & & + & & & + & + & 3 \\
\hline \multicolumn{13}{|c|}{ Differential species of the subassociation } \\
\hline Crataegus laevigata & $b$ & 2 & 4 & 1 & 1 & 2 & 2 & + & 2 & + & 2 & 5 \\
\hline Lysimachia nummularia & c & + & 1 & 2 & 2 & + & + & + & 2 & 2 & 2 & 5 \\
\hline Carex riparia & & 1 & 2 & 3 & 3 & 3 & 3 & 3 & & 2 & + & 5 \\
\hline Lycopus europaeus & & $(+)$ & + & + & + & + & 2 & 2 & + & + & & 5 \\
\hline Cardamine pratensis & & + & 2 & + & + & + & & & + & & + & 4 \\
\hline Deschampsia caespitosa & & + & + & + & + & + & & & & & & 3 \\
\hline Alisma plantago-aquatica & & & & + & + & & + & & + & $(+)$ & & 3 \\
\hline \multicolumn{13}{|l|}{ Populetalia albae* } \\
\hline Ulmus minor & a & 2 & + & + & + & 1 & & & + & & & 3 \\
\hline Populus nigra & & & & & & & 2 & & 2 & & & 1 \\
\hline Salix alba & & & & & & & & 2 & & & & 1 \\
\hline Ulmus minor & $b$ & 3 & 1 & + & + & 2 & 3 & 3 & 2 & & 3 & 5 \\
\hline Salix alba & & & & & & & & & & + & & 1 \\
\hline Carex pendula & c & 2 & 1 & 1 & 2 & 2 & 2 & & 2 & + & 2 & 5 \\
\hline Ulmus minor & & + & + & & & & + & 2 & 2 & & + & 3 \\
\hline Salix alba & & & & & & & & & & + & & 1 \\
\hline \multicolumn{13}{|l|}{ Alnetea glutinosae } \\
\hline Salix cinerea & $b$ & & & & & & & + & & & & 1 \\
\hline Valeriana dioica & c & & + & 1 & + & & & & & & & 2 \\
\hline Solanum dulcamara & & & & & & & & + & & + & & 1 \\
\hline \multicolumn{13}{|l|}{ Fagetalia } \\
\hline Acer campestre & a & & & & & + & & & & & & 1 \\
\hline Acer campestre & $b$ & + & + & + & + & & & & + & + & 2 & 4 \\
\hline Lonicera caprifolium & & + & & & + & + & & & & & & 2 \\
\hline Malus sylvestris & & & & & & & & & + & & & 1 \\
\hline Ranunculus ficaria & c & 2 & 1 & + & & 1 & & & + & & & 3 \\
\hline Circaea lutetiana & & & + & & & + & & & $(+)$ & & + & 2 \\
\hline Viola reichenbachiana & & + & & + & & & & & & & + & 2 \\
\hline Acer campestre & & & & & & & & + & & + & + & 2 \\
\hline Carex sylvatica & & & & & & & & + & & + & & 1 \\
\hline
\end{tabular}


TABLE 2. (continued) - Forests dominated by Fraxinus angustifolia.

\begin{tabular}{|c|c|c|c|c|c|c|c|c|c|c|c|c|}
\hline Querco-Fagetea & & & & & & & & & & & & \\
\hline Quercus robur & a & & & & & + & & & & & & 1 \\
\hline Ruscus aculeatus & $b$ & & & & & & & & $(+)$ & $(+)$ & $(+)$ & 2 \\
\hline Quercus robur & & & & & & & & + & & & $(+)$ & 1 \\
\hline Hedera helix & c & 2 & + & + & + & 1 & & & + & & 2 & 4 \\
\hline Quercus robur & & & & & & & & $(+)$ & & & $(+)$ & 1 \\
\hline Rhamno-Prunetea & & & & & & & & & & & & \\
\hline Cornus sanguinea & $b$ & 1 & + & + & + & + & + & 2 & 2 & + & 2 & 5 \\
\hline Prunus spinosa & & 1 & & 1 & 2 & 1 & & 2 & + & + & + & 4 \\
\hline Crataegus monogyna & & 1 & + & & + & + & + & & + & & + & 4 \\
\hline Ligustrum vulgare & & + & + & & & + & & & + & & 2 & 3 \\
\hline Euonymus europaea & & + & + & & & & & & + & $(+)$ & $(+)$ & 3 \\
\hline Rhamnus catharticus & & & & & & & & + & & + & & 1 \\
\hline Clematis vitalba & & & & & & & & & $(+)$ & & $(+)$ & 1 \\
\hline Viburnum opulus & & & & & & & & + & & & & 1 \\
\hline Molinio-Arrhenatheretea & & & & & & & & & & & & \\
\hline Leucojum aestivum & c & & + & + & + & + & & & & & & 2 \\
\hline Poa trivialis & & & & & & & & + & 2 & + & + & 2 \\
\hline Ajuga reptans & & & & + & + & & + & & & & & 2 \\
\hline Lythrum salicaria & & & & + & & & & + & + & & & 2 \\
\hline Juncus effusus & & & & & & & & & $(+)$ & + & & 1 \\
\hline Phragmiti-Caricetea elatae & & & & & & & & & & & & \\
\hline Galium palustre & c & + & 2 & 1 & 1 & & + & & 2 & + & + & 4 \\
\hline Mentha aquatica & & & & 1 & 1 & & & + & + & & & 2 \\
\hline Iris pseudacorus & & & & & 1 & + & $(+)$ & & & + & & 2 \\
\hline Lysimachia vulgaris & & & & + & & + & & & & & & 1 \\
\hline Galio-Urticetea & & & & & & & & & & & & \\
\hline Geum urbanum & c & 1 & & & & + & & & & & & 1 \\
\hline Agrostietea & & & & & & & & & & & & \\
\hline Ranunculus repens & c & & 1 & 1 & + & + & 3 & + & 2 & + & + & 5 \\
\hline Agrostis stolonifera & & & & + & + & & & & & & & 1 \\
\hline Other spp. & & & & & & & & & & & & \\
\hline Rubus caesius & $b$ & 2 & 2 & 2 & 2 & 3 & 2 & 2 & + & + & + & 5 \\
\hline Prunus cerasifera & & & & & & & & + & & + & + & 2 \\
\hline Rosa arvensis & & & & & + & & & & & & & 1 \\
\hline Sorbus torminalis & & & & & + & & & & & & & 1 \\
\hline Carex otrubae & c & & & 1 & & & $(+)$ & & & + & + & 2 \\
\hline Potentilla erecta & & & & + & + & & & & & & & 1 \\
\hline Taraxacum officinale & & & & & & + & & & + & & & 1 \\
\hline Galium aparine & & & & & & & & 2 & & & + & 1 \\
\hline Bryophyta & d & & & & & & & & & & & \\
\hline Brachythecium rutabulum & & + & & + & + & + & & + & + & + & + & 4 \\
\hline Anomodon viticulosus & & + & + & + & & & + & & + & + & + & 3 \\
\hline Neckera complanata & & & + & & & + & & & + & + & + & 3 \\
\hline Eurhynchium hians & & + & 2 & & + & & & & & & & 2 \\
\hline Calliergonella cuspidata & & & & 1 & & & & & + & + & & 2 \\
\hline Hypnum cupressiforme & & & & & + & & & + & & & + & 2 \\
\hline Campylium stellatum & & & + & & & & + & & & & & 1 \\
\hline Fissidens taxifolius & & & & & & & + & & & + & & 1 \\
\hline Homalothecium sericeum & & & & + & & & & & + & & & 1 \\
\hline Brachythecium salebrosum & & & & & & & + & + & & & & 1 \\
\hline
\end{tabular}


TABLE 3. Mixed forests dominated by Quercus robur, Fraxinus angustifolia, Ulmus minor and Carpinus betulus.

\begin{tabular}{|c|c|c|c|c|c|c|c|c|c|c|c|c|c|c|c|c|c|c|c|c|c|c|c|c|c|}
\hline Number of relevé & & 1 & 2 & 3 & 4 & 5 & 6 & 7 & 8 & 9 & 10 & 11 & 12 & 13 & 14 & 15 & 16 & 17 & 18 & 19 & 20 & 21 & 22 & 23 & \\
\hline Number in Figure 1 & & 1 & 3 & 5 & 9 & 4 & 6 & 11 & 7 & 8 & 12 & 10 & 2 & 13 & 15 & 17 & 14 & 16 & 22 & 19 & 21 & 18 & 20 & 23 & \\
\hline Altitude in $\mathrm{m}$ & & 14 & 15 & 15 & 15 & 15 & 16 & 15 & 14 & 14 & 13 & 14 & 14 & 16 & 16 & 16 & 16 & 16 & 17 & 14 & 14 & 16 & 14 & 18 & \\
\hline Releve area $\left(00 \mathrm{~m}^{2}\right)$ & & 4 & 4 & 4 & 4 & 4 & 4 & 4 & 4 & 4 & 4 & 4 & 4 & 4 & 4 & 4 & 4 & 4 & 6 & 4 & 6 & 4 & 4 & 5 & Pres. \\
\hline Cover in \%: - tree layer (a) & & 100 & 80 & 85 & 90 & 85 & 90 & 70 & 95 & 95 & 90 & 80 & 100 & 90 & 100 & 80 & 90 & 100 & 95 & 90 & 80 & 100 & 85 & 75 & deg. \\
\hline - schrub layer (b) & & 70 & 70 & 80 & 70 & 30 & 50 & 60 & 60 & 35 & 80 & 60 & 60 & 70 & 70 & 30 & 40 & 70 & 40 & 70 & 70 & 50 & 80 & 50 & \\
\hline - herb layer (c) & & 50 & 70 & 60 & 70 & 80 & 60 & 80 & 80 & 90 & 70 & 60 & 70 & 90 & 95 & 90 & 100 & 90 & 95 & 85 & 80 & 80 & 90 & 90 & \\
\hline - moos layer (d) & & 1 & 1 & 1 & 1 & 1 & 1 & 1 & 1 & 1 & 1 & 1 & 1 & 1 & 1 & 1 & 1 & 1 & 1 & 1 & 1 & 1 & 1 & 1 & \\
\hline \multicolumn{26}{|c|}{ Diagnostic species of the association } \\
\hline Ruscus aculeatus & $\mathrm{b}$ & 1 & 1 & + & + & 1 & + & 1 & 1 & 1 & + & + & 2 & 4 & 3 & 2 & 3 & 3 & 1 & 4 & 4 & 3 & 4 & 1 & 5 \\
\hline Lonicera caprifolium & & - & - & - & - & + & + & + & 1 & + & + & - & + & - & - & - & - & - & + & + & + & + & + & + & 3 \\
\hline Carex pendula & c & + & 1 & 1 & + & + & 3 & 1 & 1 & 3 & 1 & + & 2 & 1 & + & 1 & + & - & 1 & 1 & 2 & 1 & 2 & + & 5 \\
\hline Primula vulgaris & & + & + & 1 & + & 1 & + & 1 & 1 & 1 & + & + & 1 & 1 & 1 & 1 & 1 & 1 & 2 & 1 & 2 & + & 2 & 1 & 5 \\
\hline Lonicera caprifolium & & + & - & + & - & - & 1 & 1 & 1 & 1 & - & - & - & + & - & 1 & + & - & 1 & + & + & 1 & 1 & 1 & 4 \\
\hline \multicolumn{26}{|c|}{ Differential species of the subtypes } \\
\hline Prunus spinosa & $b$ & - & - & 1 & + & + & + & 1 & 2 & + & 1 & 1 & - & - & + & - & - & - & - & - & - & - & - & - & 3 \\
\hline Carex remota & c & + & 1 & + & 2 & 1 & - & 2 & + & + & 1 & 2 & + & - & - & - & - & - & - & - & - & - & + & - & 3 \\
\hline Lysimachia nummularia & & - & + & - & 1 & + & + & 1 & - & - & 1 & 1 & - & - & - & - & - & - & - & - & - & - & - & - & 2 \\
\hline Deschampsia caespitosa & & - & + & + & 1 & + & + & + & - & + & + & 1 & + & - & - & - & - & - & - & - & - & - & - & - & 3 \\
\hline Vinca minor & & - & - & - & - & - & - & - & + & 1 & - & - & 1 & 3 & 4 & 2 & 3 & 3 & 4 & 1 & 1 & 1 & 3 & 3 & 3 \\
\hline Listera ovata & & - & - & - & - & - & - & - & - & - & - & - & - & + & 1 & + & + & + & + & - & + & - & + & + & 2 \\
\hline Symphytum tuberosum & & - & - & - & - & - & - & - & - & - & - & - & - & + & 1 & - & 1 & 1 & 2 & + & - & + & - & + & 2 \\
\hline Lamium galeobdolon & & - & - & - & - & - & - & - & - & - & - & - & - & 2 & 3 & - & 2 & 3 & - & 1 & - & - & 1 & - & 2 \\
\hline Euphorbia amygdaloides & & - & - & - & - & - & - & - & - & - & - & - & - & - & - & + & + & - & + & - & + & - & 1 & + & 2 \\
\hline Polygonatum multiflorum & & - & - & - & - & - & + & - & - & - & - & - & + & - & - & - & - & 2 & + & 2 & 2 & - & 2 & - & 2 \\
\hline Pulmonaria officinalis & & - & - & - & - & - & + & - & - & - & - & - & + & - & - & - & - & - & + & - & - & + & - & 1 & 2 \\
\hline
\end{tabular}

\section{Carpinion betuli, Erythronio Carpinion}

\begin{tabular}{|c|c|c|c|c|c|c|c|c|c|c|c|c|c|c|c|c|c|c|c|c|c|c|c|c|c|}
\hline Carpinus betulus & $a$ & 1 & - & - & - & 1 & 1 & 1 & + & + & - & 1 & 1 & 2 & 3 & + & 3 & 1 & 2 & 1 & 1 & 3 & 2 & 3 & 5 \\
\hline Acer campestre & & 3 & 2 & 1 & + & 2 & 2 & 1 & - & 1 & - & + & 3 & 3 & 2 & 2 & 2 & 2 & + & 1 & 3 & 2 & 1 & 2 & 5 \\
\hline Carpinus betulus & $b$ & + & + & - & - & - & + & - & - & - & - & - & 1 & - & - & + & - & - & + & + & 1 & + & + & + & 3 \\
\hline Acer campestre & & + & + & + & 1 & 1 & + & + & + & + & - & + & + & + & + & + & + & + & + & 1 & + & + & + & + & 5 \\
\hline Tilia cordata & & - & - & - & - & - & - & - & - & - & - & - & - & + & - & - & - & - & - & - & - & - & - & - & 1 \\
\hline Carpinus betulus & c & - & - & - & - & + & - & - & - & - & - & - & - & - & - & - & + & - & + & - & - & - & - & - & 1 \\
\hline Acer campestre & & + & - & - & - & - & - & - & - & - & - & - & - & + & - & - & + & - & - & - & - & - & - & - & 1 \\
\hline Helleborus odorus & & - & - & - & - & - & - & - & - & - & - & - & - & + & - & - & - & - & + & - & - & - & - & + & 1 \\
\hline Knautia drymeia & & - & - & - & - & - & - & - & - & - & - & - & - & - & - & - & - & - & - & + & - & - & + & - & 1 \\
\hline \multicolumn{26}{|l|}{ Fagetalia } \\
\hline Sambucus nigra & $b$ & - & - & - & - & - & - & - & + & - & - & - & - & - & - & - & - & - & - & - & - & - & - & - & 1 \\
\hline Carex sylvatica & c & + & + & + & + & 1 & 2 & + & - & + & + & + & 1 & + & + & + & 1 & + & 1 & 1 & 1 & 1 & 1 & 1 & 5 \\
\hline Viola reichenbachiana & & + & + & + & + & 1 & + & + & + & - & + & + & + & + & - & + & + & + & + & - & + & + & + & - & 5 \\
\hline Circaea lutetiana & & + & + & - & + & - & + & + & - & 1 & + & - & + & + & - & - & - & - & - & - & + & - & - & + & 3 \\
\hline Brachypodium sylvaticum & & - & - & - & - & - & - & - & - & - & + & - & + & - & - & - & - & - & + & - & - & + & + & + & 2 \\
\hline Ranunculus ficaria & & - & + & + & - & - & - & - & - & 1 & - & - & - & - & - & - & - & - & - & - & - & - & - & - & 1 \\
\hline Allium ursinum & & - & - & - & - & - & - & - & - & - & - & - & - & 1 & - & - & 2 & 3 & - & - & - & - & - & - & 1 \\
\hline Euphorbia dulcis & & - & - & - & - & - & - & - & - & - & - & - & - & + & - & - & - & - & + & - & - & - & - & + & 1 \\
\hline Lathyrus vernus & & - & - & - & - & - & - & - & - & - & - & - & - & - & - & - & - & - & + & - & - & - & - & - & 1 \\
\hline Salvia glutinosa & & - & - & - & - & - & - & - & - & - & - & - & - & - & - & - & - & - & 1 & - & - & - & - & - & 1 \\
\hline Mercurialis perennis & & - & - & - & - & - & - & - & - & - & - & - & - & - & - & - & - & - & - & - & 2 & - & - & - & 1 \\
\hline \multicolumn{26}{|l|}{ Populetalia albae } \\
\hline Fraxinus angustifolia & a & 3 & 4 & 4 & 4 & 3 & 4 & 5 & 3 & 2 & 3 & 4 & 2 & + & 2 & 2 & 2 & + & 2 & + & + & 2 & 2 & + & 5 \\
\hline Ulmus minor & & - & 1 & 1 & 3 & 1 & 1 & - & 1 & 1 & 1 & 1 & - & + & - & + & - & - & + & 2 & + & - & 1 & 1 & 4 \\
\hline Ulmus minor & $b$ & + & 1 & 1 & 2 & 1 & + & + & + & + & 1 & 1 & + & + & - & - & + & - & + & 1 & + & + & 1 & 1 & 5 \\
\hline Fraxinus angustifolia & & + & - & + & 1 & - & + & + & - & 1 & + & + & + & + & - & - & - & + & - & + & + & + & - & + & 4 \\
\hline Ranunculus lanuginosus & c & 2 & 2 & 1 & + & 1 & 1 & 1 & - & 1 & + & + & - & + & + & + & + & - & - & - & - & - & - & - & 3 \\
\hline Rumex sanguineus & & - & - & - & + & - & - & + & - & + & + & - & - & - & - & - & - & - & - & - & - & - & - & - & 1 \\
\hline Fraxinus angustifolia & & - & - & - & + & - & - & + & - & - & - & - & - & - & - & - & - & - & + & - & - & - & - & - & 1 \\
\hline Ulmus minor & & + & - & - & - & - & - & - & - & - & - & - & - & - & - & - & - & - & - & - & - & - & - & - & 1 \\
\hline
\end{tabular}


TABLE 3. (continued) - Mixed forests dominated by Quercus robur, Fraxinus angustifolia, Ulmus minor and Carpinus betulus.

\begin{tabular}{|c|c|c|c|c|c|c|c|c|c|c|c|c|c|c|c|c|c|c|c|c|c|c|c|c|c|}
\hline \multicolumn{26}{|l|}{ Alnetalia glutinosae } \\
\hline Lycopus europaeus & c & - & - & - & - & - & - & + & - & - & + & + & - & - & - & - & - & - & - & - & - & - & - & - & 1 \\
\hline Cardamine pratensis & & + & - & + & - & - & - & - & - & - & - & - & - & - & - & - & - & - & - & - & - & - & - & - & 1 \\
\hline \multicolumn{26}{|l|}{ Querco-Fagetae } \\
\hline Quercus robur & $a$ & 4 & 1 & 1 & 1 & 1 & + & 1 & 2 & 3 & 3 & 2 & 4 & 3 & 3 & 3 & 2 & 4 & 3 & 4 & 4 & 3 & 3 & + & 5 \\
\hline Pyrus communis & & - & - & - & - & - & - & - & - & - & - & - & - & - & - & - & - & - & + & - & - & + & - & - & 1 \\
\hline Corylus avellana & & - & - & - & - & - & - & - & - & - & - & - & - & - & - & - & - & + & - & - & - & - & - & - & 1 \\
\hline Corylus avellana & b & - & + & 1 & + & 1 & + & - & - & + & + & + & + & 1 & 1 & 1 & 1 & 1 & 1 & 1 & 2 & 2 & 1 & + & 5 \\
\hline Quercus robur & & 1 & + & - & + & - & + & - & - & + & + & - & + & - & + & - & + & + & - & + & + & - & - & - & 3 \\
\hline Pyrus communis & & - & - & - & - & - & - & - & - & + & - & - & - & - & - & - & - & - & - & - & - & - & - & - & 1 \\
\hline Hedera helix & c & 3 & 3 & 3 & 2 & 3 & 2 & 3 & 4 & 4 & 3 & + & 3 & 1 & 2 & 3 & 1 & 1 & 3 & 3 & 4 & 3 & 3 & 4 & 5 \\
\hline Quercus robur & & + & + & + & + & - & + & - & - & - & - & - & + & + & + & 1 & - & + & + & - & 1 & + & + & + & 4 \\
\hline Arum italicum & & - & - & - & - & + & - & - & + & - & - & - & - & + & + & + & - & - & + & - & - & - & - & - & 2 \\
\hline Tamus communis & & - & - & - & - & - & - & - & + & + & - & - & - & - & + & - & - & - & - & + & 1 & - & + & - & 2 \\
\hline Anemone nemorosa & & - & - & - & - & - & - & - & - & - & - & - & - & - & - & - & + & 2 & - & - & - & - & - & - & 1 \\
\hline \multicolumn{26}{|l|}{ Rhamno-Prunetea } \\
\hline Crataegus oxyacantha & $b$ & 3 & 2 & 4 & 2 & 1 & 2 & 1 & + & 1 & 4 & 3 & 3 & + & 1 & + & - & + & 1 & 1 & 2 & 1 & 1 & 3 & 5 \\
\hline Crataegus monogyna & & - & 1 & + & + & + & + & + & + & + & - & + & + & + & + & + & 1 & + & + & + & + & - & + & + & 5 \\
\hline Cornus sanguinea & & - & 1 & - & + & + & + & - & + & + & + & + & + & + & - & + & + & + & 2 & + & + & - & 1 & 1 & 4 \\
\hline Ligustrum vulgare & & 1 & + & + & - & 1 & 1 & 1 & 2 & 2 & - & - & 1 & - & - & + & + & + & 1 & 1 & 1 & + & + & 1 & 4 \\
\hline Viburnum opulus & & + & + & + & - & - & + & - & + & 1 & 1 & + & + & - & - & + & - & + & + & + & + & + & + & - & 4 \\
\hline Euonymus europaea & & + & + & + & + & - & - & - & + & + & + & - & - & + & + & + & - & - & - & + & - & - & - & - & 3 \\
\hline Rhamnus cathartica & & - & + & - & - & + & - & - & + & + & - & - & - & - & + & + & - & - & - & - & - & - & + & - & 2 \\
\hline Prunus ceracifera & & - & - & - & - & - & - & - & - & - & + & - & - & - & - & - & - & - & - & - & - & + & - & - & 1 \\
\hline Ligustrum vulgare & c & - & + & - & - & - & - & - & 1 & - & + & - & - & - & - & - & - & - & + & - & - & + & - & - & 1 \\
\hline Rubus plicatus & & - & - & - & - & - & - & - & - & - & + & - & - & - & - & - & - & - & - & - & - & - & - & - & 1 \\
\hline
\end{tabular}

\section{Molinio-Arrhenatheretea}

Ajuga reptans

Lythrum salicaria

Prunella vulgaris

\section{Other species}

\section{Rosa arvensis}

Cornus mas

Eupatorium cannabinum

Laurus nobilis

Rubus caesius

Aegopodium podagraria

Ranunculus repens

Potentilla erecta

Galium palustre

Vicia dumetorum

Glechoma hederacea

Fragaria vesca

Ophioglossum vulgatum

Viola hirta

Polypodium vulgare

Erigeron annuus

Plantago major

Stellaria nemorum

Equisetum telmateia

\section{Bryophyta}

\section{Anomodon viticulosus}

Brachythecium rutabulum

Fissidens taxifolius

Neckera complanata

Eurhynchium hians

Homalothecium sericeum

Brachythecium salebrosum

Calliergonella cuspidata

Hypnum cupressiforme

Leucodon sciuroides

Campylium stellatum

Hygroamblystegium tenax 
TABLE 4. Differential species (marked grey) with frequency ( $\geq 30 \%$ ) and fidelity (phi coefficient $\times 100, \geq 35$ ) values for the compared mixed hardwood syntaxa: (1) Genisto eletae-Quercetum roboris, [57, 46, 47, 58, 50], N-Croatia; (2) Carpino betuliQuercetum roboris, [54, 47, 51, 56, 50], N-Croatia; (3) 23 relevés in the present study + 12 relevés Bertović [2], (4) Fraxino pannonicae-Quercetum roboris, [31, 32], Italy.

\begin{tabular}{|c|c|c|c|c|}
\hline Group No. & 1 & 2 & 3 & 4 \\
\hline No. of relevés & 74 & 87 & 35 & 20 \\
\hline \multicolumn{5}{|l|}{ Diagnostic species } \\
\hline Iris pseudacorus & $75^{76.5}$ & $9-$ &.- & -- \\
\hline Genista tinctoria & $70^{72.7}$ & $9-$ &.-- &.- \\
\hline Stachys palustris & $58^{67.1}$ & $5^{--}$ &.-- & -- \\
\hline Caltha palustris & $52^{66.9}$ &.-- &.-- &.-- \\
\hline Carex strigosa & $62^{66.8}$ & $9--$ &.- & $\cdot--$ \\
\hline Lycopus europaeus & $82^{64.0}$ & $19--$ & $22-$ &.-- \\
\hline Mentha aquatica & $52^{62.3}$ &.- &.-- & $5^{--}$ \\
\hline Glechoma hederacea & $83^{59.5}$ & $51^{20.5}$ & $3^{--}$ &.- \\
\hline Leucojum aestivum & $43^{58.9}$ & $1^{--}$ & $-\cdot-$ &.-- \\
\hline Carex vesicaria & $39^{56.9}$ &.-- &.- &.- \\
\hline Carex elongata & $43^{56.8}$ & $3^{--}$ &.- & -- \\
\hline Galium palustre & $88^{54.5}$ & $18^{--}$ & $11^{--}$ & $50^{9.6}$ \\
\hline Lysimachia vulgaris & $61^{54.4}$ & $3^{--}$ & $14^{--}$ & $10^{--}$ \\
\hline Carex vulpina & $38^{52.8}$ &.-- & $3^{--}$ &.-- \\
\hline Lythrum salicaria & $49^{52.0}$ & $2^{--}$ & $8^{--}$ & $5^{--}$ \\
\hline Euphorbia palustris & $32^{51.5}$ &.-- &.-- &.-- \\
\hline Ranunculus repens & $82^{50.0}$ & $16^{--}$ & $31^{--}$ & $30^{--}$ \\
\hline Solanum dulcamara & $39^{49.5}$ & $7^{--}$ &.- &.- \\
\hline Lychnis flos-cuculi & $52^{49.1}$ & $3^{--}$ &.-- & $20^{1.8}$ \\
\hline Mentha verticillata agg. & $30^{45.4}$ & $3-$ &.-- &.-- \\
\hline Peucedanum palustre & $30^{45.4}$ & $3^{--}$ &.-- & -- \\
\hline Myosotis palustris agg. & $36^{45.2}$ & $9--$ &.-- &.- \\
\hline Lysimachia nummularia & $74^{40.4}$ & $41^{1.0}$ & $44^{5.5}$ &.- \\
\hline Cardamine pratensis & $44^{38.1}$ & $9--$ & $6^{--}$ & $15^{--}$ \\
\hline Cerastium sylvaticum & $43^{36.4}$ & $28^{14.4}$ & $3^{--}$ &.- \\
\hline Oxalis acetosella &.- & $48^{63.9}$ &.-- &.-- \\
\hline Galium odoratum &.- & $42^{59.1}$ &.-- & -- \\
\hline Dryopteris filix-mas & $3^{--}$ & $42^{56.3}$ &.-- &.- \\
\hline Athyrium filix-femina & $21^{2.6}$ & $55^{53.3}$ &.-- & -- \\
\hline Tilia cordata &.- & $38^{52.6}$ & $3^{--}$ &.-- \\
\hline Veronica montana & $12^{--}$ & $68^{52.3}$ &.-- & $30^{3.4}$ \\
\hline Galeobdolon luteum & $1^{--}$ & $50^{48.9}$ & $19^{2.7}$ &.-- \\
\hline Geum urbanum & $19^{4.9}$ & $46^{46.1}$ &.-- &.-- \\
\hline Carex brizoides & $6^{--}$ & $32^{44.1}$ &.-- &.- \\
\hline Anemone nemorosa &.- & $33^{43.3}$ & $8^{--}$ &.- \\
\hline Veronica chamaedrys & $6^{--}$ & $35^{42.0}$ & $6^{--}$ &.- \\
\hline Primula vulgaris &.- & $11^{--}$ & $97^{90.9}$ &.-- \\
\hline Lonicera caprifolium &.- & $3^{--}$ & $81^{69.3}$ & $25^{--}$ \\
\hline Carex pendula & $4^{--}$ & $17^{--}$ & $97^{68.3}$ & $40^{--}$ \\
\hline Vinca minor &.-- & $4^{--}$ & $53^{63.7}$ & -- \\
\hline Ranunculus lanuginosus &.-- & $5^{--}$ & $53^{55.0}$ & $10^{--}$ \\
\hline
\end{tabular}


TABLE 4. (continued) - Differential species (marked grey) with frequency ( $\geq 30 \%$ ) and fidelity (phi coefficient $\times 100, \geq 35$ ) values for the compared mixed hardwood syntaxa: (1) Genisto eletae-Quercetum roboris, [57, 46, 47, 58, 50], N-Croatia; (2) Carpino betuli-Quercetum roboris, [54, 47, 51, 56, 50], N-Croatia; (3) 23 relevés in the present study +12 relevés Bertović [2], (4) Fraxino pannonicae-Quercetum roboris, [31, 32], Italy.

\begin{tabular}{|c|c|c|c|c|}
\hline Group No. & 1 & 2 & 3 & 4 \\
\hline No. of relevés & 74 & 87 & 35 & 20 \\
\hline \multicolumn{5}{|l|}{ Diagnostic species } \\
\hline Ligustrum vulgare & $1^{--}$ & $17^{--}$ & $89^{68.7}$ & $25^{--}$ \\
\hline Corylus avellana & $4^{--}$ & $31^{2.1}$ & $83^{67.9}$ &.-- \\
\hline Ruscus aculeatus &.-- & $27^{--}$ & $97^{64.1}$ & $45^{3.1}$ \\
\hline Viburnum opulus & $22^{--}$ & $19--$ & $78^{60.8}$ &.- \\
\hline Aegopodium podagraria & $16^{--}$ & $17^{--}$ & $64^{53.8}$ &.-- \\
\hline Listera ovata &.- & $16^{--}$ & $47^{50.0}$ &.- \\
\hline Cornus sanguinea & $14^{--}$ & $33^{--}$ & $81^{46.7}$ & $35-$ \\
\hline Symphytum tuberosum agg. &.-- & $12-$ & $36^{42.3}$ &.- \\
\hline Arum italicum &.-- &.-- & $28^{41.2}$ & $5^{--}$ \\
\hline Iris foetidissima &.-- &.-- &.-- & $65^{76.3}$ \\
\hline Rubus ulmifolius &.-- &.-- &.-- & $60^{72.8}$ \\
\hline Luzula forsteri &.-- & $2-$ &.-- & $60^{70.9}$ \\
\hline Moehringia trinervia & $8^{--}$ & 18 &.-- & $65^{58.5}$ \\
\hline Myosotis sylvatica &.-- &.-- &.-- & $40^{57.7}$ \\
\hline Quercus ilex &.-- &.-- &.-- & $40^{57.7}$ \\
\hline Holcus lanatus &.-- &.-- &.-- & $35^{53.6}$ \\
\hline Brachypodium sylvaticum & 3 & $39^{6.5}$ & $17^{--}$ & $75^{51.2}$ \\
\hline Cyclamen repandum &.-- &.-- &.-- & $30^{49.3}$ \\
\hline Hypericum androsaemum &.-- &.-- &.-- & $30^{49.3}$ \\
\hline Populus alba & $4^{--}$ &.-- &.-- & $35^{49.3}$ \\
\hline Pteridium aquilinum &.-- & $2-$ & $3-$ & $30^{43.6}$ \\
\hline Veronica officinalis & $4^{--}$ & $3-$ &.-- & $30^{41.3}$ \\
\hline \multicolumn{5}{|c|}{ Diagnostic species for more syntaxa } \\
\hline Persicaria hydropiper & $31^{28.5}$ & $25^{18.2}$ &.-- &.-- \\
\hline Frangula alnus & $34^{26.6}$ & $19^{3.3}$ & $14^{--}$ &.-- \\
\hline Agrostis stolonifera & $44^{28.9}$ & $3^{--}$ &.-- & $45^{30.1}$ \\
\hline Poa trivialis & $44^{28.0}$ & $5^{--}$ &.-- & $45^{29.1}$ \\
\hline Juncus effusus & $48^{25.2}$ & $18^{--}$ & $3^{--}$ & $45^{21.3}$ \\
\hline Urtica dioica & $43^{16.5}$ & $31^{1.9}$ &.-- & $45^{19.2}$ \\
\hline Carpinus betulus & $17^{--}$ & $100^{47.0}$ & $89^{33.8}$ & $35^{--}$ \\
\hline Euphorbia amygdaloides &.-- & $28^{14.2}$ & $36^{26.1}$ & $10^{--}$ \\
\hline Polygonatum multiflorum &.-- & $34^{26.9}$ & $33^{25.3}$ &.-- \\
\hline Pyrus pyraster & $18^{--}$ & $25^{8.6}$ & $33^{20.9}$ &.- \\
\hline Pulmonaria officinalis & $1^{--}$ & $15^{5.4}$ & $31^{34.1}$ &.-- \\
\hline Crataegus monogyna & $36^{--}$ & $60^{4.0}$ & $86^{34.0}$ & $45^{--}$ \\
\hline Crataegus laevigata & $35^{--}$ & $70^{23.9}$ & $92^{49.1}$ &.- \\
\hline Acer campestre & $29--$ & $89^{23.5}$ & $97^{34.5}$ & $65^{--}$ \\
\hline Viola reichenbachiana & $12^{--}$ & $70^{15.0}$ & $86^{34.1}$ & $60^{3.6}$ \\
\hline Carex sylvatica & $9--$ & $68^{7.9}$ & $97^{42.9}$ & $70^{10.6}$ \\
\hline Euonymus europaeus & $10^{--}$ & $62^{22.5}$ & $50^{7.9}$ & $50^{7.9}$ \\
\hline Hedera helix & $9--$ & $49--$ & $100^{44.0}$ & $95^{38.0}$ \\
\hline Carex flacca &.- &.- & $28^{28.2}$ & $20^{14.3}$ \\
\hline
\end{tabular}


were classified in the alliance Erythronio-Carpinion, and more precisely, according to the latest phytogeographical differentiation of oak-hornbeam forests of southeast Europe [19], they are classified in the sub-alliance Lonicero caprifoliaeCarpinenion betuli group Quercus robur, within the alliance Erythronio-Carpinion. In the studied mixed hardwood forests, of the species characteristic of the alliance abundantly present were Primula vulgaris and Lonicera caprifolium, while of the differential species of the sub-alliance these are Anemone nemorosa, Vinca minor, Euphorbia dulcis, Knautia drymeia and Lamium galeobdolon, and of the differential species of the group Quercus robur these are Quercus robur, Carex remota and Circaea lutetiana (cf. Košir et al. [19], Table 1).

Although in the dendrogram on Figure 3, the continental oak-hornbeam forests and the studied stands in Istria are separated at a relatively low level, they cannot be considered as belonging to the same association. The mixed hardwood forests of the Mediterranean area contain a certain number of species from the warmer climate, but also a higher presence of the narrow-leaved ash, European white elm, and some species associated with a high level of ground waters. They lack a part of continental species that do not penetrate wet habitats of the Mediterranean area, and are frequent in the continental forests of the common oak and common hornbeam (for example, Galium odoratum, Oxalis acetosella, Dryopteris filix-mas, Tilia cordata and others). In addition, continental stands are described in the association Lonicero caprifoliae-Quercetum roboris [45], which is broadly understood and of heterogeneous composition. This was also confirmed by Douda et al. [10], when they divided it into associations Fraxino panonnicae-Ulmetum glabrae and Ficario vernae-Ulmetum campestris, within the alliance Alnion incanae. However, the majority of stands of the association Lonicero caprifoliae-Quercetum roboris belong to the snytaxa within the Carpinion alliance: stands with the species of Illyrian floristic geo-element will be classified within the alliance Erythronio-Carpinion, and those in which these species are lacking in the alliance Carpinion betuli. The center of distribution and the largest areas of this association are located in Pannonian Croatia, where they are known under the name Carpino betuli-Quercetum roboris [46, 47], whereas in Hungary similar forests have been described as association Circaeo lutetianae-Quercetum roboris and others [48].

On the basis of previous vegetation research and this analysis, we have classified mixed forests of hardwood broadleaved trees along the Mirna River in the association Asparago-tenuifolii-Quercetum roboris. Its area is in the Mediterranean region of the north-western part of Slovenia and Croatia, and in the eastern part of Venezia Giulia. The diagnostic species of the association are Ruscus aculeatus, Lonicera caprifolium, Carex pendula, Primula vulgaris and Arum italicum. Although it is of extrazonal character, the cover of the species of the Illyrian floral geoelement is high. The subMediterranean climate benefits their relatively thermophilic character.

The species Asparagus tenuifolius is absent from our phytocenological relevés, but is abundantly present in the surrounding zonal forests of the downy oak and the European hop-hornbeam. It does not respond well to wet and occasionally flooded habitats.

Future research of forests in the river valleys of the North
Adriatic littoral will show the justifiability of classifying the studied forest stands of hardwood broadleaved trees into the association Asparago tenuifoli-Quercetum roboris and the alliance Erythronio-Carpinion betuli. Based on our analyses and recent phytocenological trends, their separation into new syntaxa is for now unfounded.

The association Asparago tenuifolii-Quercetum roboris was studied in two larger localities, hence its analysis in the hierarchical phytocoenological system is not yet warranted. The study of the stand along the Mirna River can be divided into two subtypes (Table 3): the first subtype of 12 relevés (subtype Carex remota, relevés 1-12 in Table 3) is distributed on wetter habitat, shallow depressions and very mild slopes, and according to the drier subtype, the prominent differential species are Carex remota, Deschampsia cespitosa, Prunus spinosa, Lysimachia nummularia, Ranunculus lanuginosus. In addition to them, there is also an increased presence of other hygrophilous species, especially Ulmus minor.

Relevés 13-23 in Table 3 represents a drier subtype (subtype Vinca minor) of mixed hardwood stands especially distributed on highest terrains along the Mirna River. The differential species according to the first subtype are Vinca minor, Listera ovata, Symphytum tuberosum, Galeobdolon luteum, Euphorbia amygdaloides, Polygonatum multiflorum and Pulmonaria officinalis. To a lesser extent, other species of less humid habitats also have diagnostic significance, and in relation to the previous subtype there is a higher presence of the common oak and common hornbeam.

It should be emphasized that the studied hardwood forests along the Mirna River represent a permanent vegetation stage conditioned by the hydrological regime, primarily by groundwater. For that reason, the presence of the species Ulmus minor and Fraxinus angustifolia is higher than in other Carpinetum communities of southeast Europe.

\section{CONCLUSIONS}

The phytocoenological study of periodically flooded and wet forests along the Mirna River in Istria and their comparisons with related syntaxa resulted in defining two associations. Fraxinus angustifolia forests with numerous hygrophilous species are classified in the association Carici remotae-Fraxinetum oxycarpae with the new sub-association crataegetosum laevigatae. The mixed hardwood forests belong to the association Asparago tenuifolii-Quercetum roboris distributed in the planar zone in broader river lowlands of the central part of the northern Mediterranean area. Both associations in the studied area lack numerous termophilous species from related syntaxa of southern Europe, as well as many hygrophilous species from related continental flooded and wet forests. The reasons primarily lie in the biogeographical position, ecological conditions and anthropogenic influences in the studied area. It is located at the northern most part of the Mediterranean, deeply retracted into the European continent. It is also marked by Mediterranean and continental climate impacts, especially modified by the $15 \mathrm{~km}$ distant Dinaric mountains with numerous species of the Illyrian floristic geoelement. These reasons have caused a certain isolation and transitional character of the area, which, in turn, has reflected on the floristic composition of the studied forests. 
Syntaxonomical scheme:

Class: Querco roboris-Fagetea sylvaticae Br.-Bl. et Vlieger in Vlieger 1937

Order: Populetalia albae Br.-Bl. ex Tchou 1949

Alliance: Carici remotae-Fraxinion oyycarpae Pedrotti ex Pedrotti, Biondi, Allegrezza \& Casavecchia in Biondi et al. 2014

Ass: Carici-Fraxinetum oxycarpae Pedrotti 1970 ex 1992

crataegetosum laevigatae subass. nova hoc. loco

Order: Fagetalia Pawlowski in Pawlowski et al. 1928

Alliance: Erythronio-Carpinion betuli (Horvat 1938) Marinček in Wallnöfer et al. 1993

Suballiance: Lonicero caprifoliae-Carpinenion betuli Vukelić in Marinček 1994 group Quercus robur

Ass: Asparago tenuifolii-Quercetum roboris (Lausi 1967) Marinček 1994

Carex remota subtype

Vinca minor subtype

The study results add to the knowledge of the composition and character of flooded and wet forests in this part of Europe, and will also serve to resolve the issues with their syntaxonomy. In addition, they are important in the preservation of these rare and endangered habitat types.

\section{Acknowledgements}

This work has been fully supported by the Croatian Science Foundation under the project number IP-2014-09-1834.

\section{REFERENCES}

1. HORVAT I, GLAVAČ V, ELLENBERG H 1974 Vegetation Südosteuropas. Gustav Fischer Verlag, Stuttgart, Germany, $768 \mathrm{p}$

2. BERTOVIĆ S 1975 The Mirna River Valley and Motovun Forest in Istria (Croatia). Phytocoenologia 2 (3/4): 329-335

3. BERTOVIĆ S, LOVRIĆ AŽ 1987 Šumske zajednice Jugoslavije, SR Hrvatska. In: Potočić Z (ed) Šumarska enciklopedija, Vol 3, 2nd Edition. Leksikografski zavod Miroslav Krleža, Zagreb, Croatia, pp 395-404

4. MARINČEK L, ČARNI A 2000 Die Unterverbände der Hainbuchenwälder des Verbandes Erythronio-Carpinion betuli (Horvat 1938) Marinček in Wallnöfer, Mucina et Grass 1993. Scopolia 45: 1-20

5. BRULLO S SPAMPINATO G 1999 Syntaxonomy of hygrophilous woods of the Alno-Quercion roboris. Annali di Botanica 57: 133-146

6. TRINAJSTIĆ | 2008 Biljne zajednice Republike Hrvatske. Akademija šumarskih znanosti, Zagreb, Croatia, $179 \mathrm{p}$

7. VUKELIĆ J 2012 Šumska vegetacija Hrvatske. Šumarski fakultet Sveučilište u Zagrebu, Zagreb, Croatia, $403 p$

8. AMIGO J, IZCO J, ROMERO MI 2004 Swamp alder woodlands in Galicia (NWSpain): phytosociological interpretation. Ecological and floristic contrast to western European swamp woodlands and delimitation versus riparian alder woodlands in southern Europe and northern Africa. Phytocoenologia 34: 613-638. DOI: https://doi.org/10.1127/0340-269X/2004/0034-0613

9. BIONDI E, BLASI C, ALLEGREZZA M, ANZELLOTTI I, AZZELLA MM, CARL E, CASAVECCHIA S, COPIZ R, et al. 2014 Plant communities of Italy: the Vegetation Prodrome. Plant Biosyst 148 (4): 728-814. DOI: https://doi.org/10.1080/11263504.20 14.948527

10. DOUDA J, BOUBLIK K, SLEZAK M, BIURRUN I, NOCIAR J, HAVRDOVA A, DOUDOVÁ J, AĆIĆ S, et al. 2016 Vegetation classification and biogeography of European floodplain forests and alder carrs. Appl Veg Sci 19 (1): 147-163. DOI: https://doi. org/10.1111/avsc.12201

11. BIURRUN I, CAMPOS JA, GARCÍA-MIJANGOS I, HERRERA M, LOIDI J 2016 Floodplain forests of the Iberian Peninsula: Vegetation classification and climatic features. App/ Veg Sci 19 (2): 336-354. DOI: https://doi.org/10.1111/avsc.12219
12. MUCINA L, BULTMANN H, DIERSEN K, THEURILLAT JP, RAUS T, ČARNI A, ŠUMBEROVÁ K, WILLNER W, et al. 2016 Vegetation of Europe: Hierarchical floristic classification system of vascular plant, bryophyte, lichen, and algal communities. Appl Veg Sci 19 (S1): 3-264. DOI: https://doi.org/10.1111/avsc.12257

13. BLASI C, CAPOTORTI G, COPIZ R, GUIDA D, MOLLO B, SMIRAGLIA D, ZAVATTERO L 2014 Classification and mapping of the ecoregions of Italy. Plant Biosyst 148 (6): 1255-1345. DOI: https://doi.org/10.1080/11263504.2014.985756

14. BRAUN-BLANQUET J 1964 Pflanzensoziologie. Grundzüge der Vegetationskunde [Plant sociology. Basic course of Vegetation Science]. Springer-Verlag, Wien, Austria, $865 p$

15. OIKON 2015 Plan restauracije starog korita rijeke Mirne. Oikon d.o.o., Zagreb, Croatia

16. NIKOLIĆ T 2004 Flora Croatica database. URL: http://hirc. botanic.hr/fcd (20 February 2018)

17. ATHERTON I, BOSANQUET S, LAWLEY M (ed) 2010 Mosses and liverworts of Britain and Ireland a field guide. British Bryological Society \& Latimer Trend \& Co. Ltd, Plymouth, United Kingdom, $848 p$

18. BIONDI E, ALLEGREZZA M, CASAVECCHIA S, GALDENZI D, GASPARRI R, PESARESI S, VAGGE I, BLASIET C 2014 New and validated syntaxa for the checklist of Italian vegetation. Plant Biosyst 148 (2): 318-332. DOI: https://doi.org/10.1080/11263 504.2014.892907

19. KOŠIR P, CASAVECCHIA S, ČARNI A, ŠKVORC Ž, ZIVKOVIC L, BIONDI E 2013 Ecological and phytogeographical differentiation of oak-hornbeam forests in southeastern Europe. Plant Biosyst 147 (1): 84-98. DOI: https://doi.org/10 $.1080 / 11263504.2012 .717550$

20. WEBER HE, MORAVEC J, THEURILLAT JP 2000 International Code of Phytosociological Nomenclature $3^{\text {th }}$ Ed. J Veg Sci 11 (5): 739-768. DOI: https://doi.org/10.2307/3236580

21. MATUSZKIEWICZ W, MATUSZKIEWICZ A 1981. Das Prinzip der mehrdimensionalen Gliederung der Vegetationseinheiten, erläutert am Beispiel der Eichen Hainbuchenwälder in Polen. In: Dierschke H (ed) Syntaxonomie. Cramer, Vaduz, Liechtenstein, pp 123-148

22. HENNEKENS SM, SCHAMINÉE JHJ 2001 TURBOVEG, a comprehensive data base management system for vegetation data. J Veg Sci 12 (4): 589-591. DOI: https://doi. org/10.2307/3237010 
23. R CORE TEAM 2016 R: A language and environment for statistical computing. R Foundation for Statistical Computing, Vienna, Austria. URL: http://www.R-project.org/

24. OKSANEN J, BLANCHET FG, FRIENDLY M, KINDT R, LEGENDRE P, MCGLINN D, MINCHIN PR, O'HARA RB, et al. 2017 Package 'Vegan': Community ecology package. Version 2.4-2. URL: https://cran.r-project.org, https://github.com/vegandevs/ vegan.

25. TICHÝ L 2002 JUICE 6.3, software for vegetation classification. J Veg Sci 13 (3): 451-453. DOI: https://doi.org/10.1658/11009233(2002)013[0451:JSFVC]2.0.CO;2

26. WORLDWIDE BIOCLIMATIC CLASSIFICATION SYSTEM, 19962017, Rivas-Martínez S, Rivas-Saenz S, Phytosociological Research Centre, Spain. URL: http://www.globalbioclimatics

27. PEDROTTI F 1970 Un relito di bosco planiziare a Quercus robur e Fraxinus angustifolia a lungo il fiume Sinello in Abruzzo. Savini-Mercuri, Camerino, Italy, $23 \mathrm{p}$

28. PEDROTTI F 1992 Tipificazione e correzione dell'associazione Carici-Fraxinetum angustifoliae Pedrotti 1970. Doc Phytosoc 14: $165-166$

29. PEDROTTI F, CORTINI PEDROTTI C 1978 Notize sulla distribuzione del Carici-Fraxinetum angustifoliae lungo la costa adriatica (Italia centro-meridionale). Mitteil Ostalp-dinar Ges Vegetationsk 14: 255-261

30. PEDROTTI F 1984 Foreste ripariali lungo la costa adriatica dell'Italia. Col loq Phytosoc (Les fôrets alluviales) 9: 143-154

31. GELLINI R, PEDROTTI F, VENANZONI R 1986 Le associazioni forestali ripariali e palustri della Selva di San Rossore (Pisa). Doc Phytosoc 10 (2): 27-42

32. MANZI A 1992 | boschi ripariali lungo il Fiume Osento (Abruzzo-Italia centrale). Doc Phytosoc 14: 115-121

33. CONTI F, PIRONE G 1992 Le cenosi di Fraxinus oxycarpa Bieb. e di Carpinus betulus L. del bosco di Vallaspra nel bacino del Fiume Sangro (Abruzzo, Italia). Doc Phytosoc 14: 167-175

34. PEDROTTI F, GAFTA D 1996. Ecologia delle foreste ripariali e paludose dell'Italia. Università degli Studi di Camerino, Camerino, Italy, $165 \mathrm{p}$

35. BRULLO S, SPAMPINATO G 1997 Indagine fitosociologica sulle ripisilve della Calabria. Lazaroa 18: 105-151

36. KAVGACCI A, ČARNI A, TECIMEN B, OZALP G 2010 Diversity and ecological differentiation of oak forest in NW Tharce (Turkey). Arch Biol Sci 62 (3): 705-718. DOI: https://doi.org/10.2298/ ABS1003705K

37. MERCADAL G, VILAR L 2013 Caracterització de les freixenedes alluvials inundables delnord-est de Catalunya (Carici remotaeFraxinetum oxycarpae Pedrotti 1970 corr. Pedrotti 1992). Orsis 27: 53-94

38. TCHOU YT 1948 Études écologiques et phytosociologiques sur les forêts riveraines du Bas-Languedoc. Vegetatio 1 (1): 2-28

39. KÁRPÁTI I, KÁRPÁTI V 1961 Die zönologischen verhältnisse der Auenwälder. Acta Bot Acad Sci Hung 7: 235-301

40. RIVAS-MARTíNEZ S, COSTA M, CASTROVIEJO S, VALDES E 1980 La vegetación de Doñana (Huelva, España). Lazaroa 2: 5-190

41. RIVAS-MARTÍNEZ S, DÍAZ TE, FERNÁNDEZ-GONZÁLEZ F, IZCO J, LOIDI J, LOUSA M, PENAS A 2002 Vascular plant communities of Spain and Portugal, addenda to the syntaxonomical checklist of 2001. Itinera Geobot 15 (1): 5-432
42. GESTI PERICH J, FONT GJ, VILAR L 2003 Rusco aculeatiFraxinetum angustifoliae, una nova associació forestal de ribera del territori ruscínic. Acta Bot Barcinon 48: 57-66

43. AMIGO J, PULGAR I, IZCO J 2009 Evidence of riverside ash tree forests in southern Galicia (NW Spain). Lazaroa 30: 181-189

44. LAUSI D 1967 Zur Klimax-frage der friaulischen Ebene. Mitt Ost-Din Pflanzensoz Arbeit 7: 41-46

45. MARINČEK L 1994 Zur Nomenklatur der Hainbuchenwälder des Erythronio-Carpinion. In: Trinajstić I (ed) Simpozij Pevalek, Flora i vegetacija Hrvatske, Proceedings, Koprivnica, Croatia, 20-22 May 1993. Faculty of Forestry University of Zagreb \& Croatian Forests Itd, Zagreb, Croatia, pp 57-62

46. RAUŠ Đ 1973 Fitocenološke značajke i vegetacijska karta fakultetskih šuma Lubardenik i Opeke. Sumar List 97 (5-6): 190-221

47. RAUŠ Đ 1975 Vegetacijski i sinekološki odnosi šuma u bazenu Spačva. Glas Šum Pokuse 18: 225-344

48. BORHIDI A, KEVEY B, LENDVAI G 2012 Plant communities of Hungary. Akadémiai Kiadó, Budapest, Hungary, $544 \mathrm{p}$

49. GLAVAČ V 1959 O šumi poljskog jasena s kasnim drijemovcem (Leucoieto-Fraxinetum angustifoliae ass. nova). Sumar List 83 (1-3): 39-45

50. BARIČEVIĆ D 1998 Ecological-vegetation characteristics of Žutica forest (in Croatian). MSc Thesis, University of Zagreb, Faculty of Forestry, Zagreb, Croatia, $154 \mathrm{p}$

51. ŠKVORC Ž, CESTARIĆ D, FRANJIĆ J, KRSTONOŠIĆ D, SEVER K GUZMIĆ M 2009 Forest vegetation dynamics of the Spačva basin in the past forty years (in Croatian with English summary). In: Matić S, Anić I (eds) Forests of pedunculate oak in changed site and management conditions, Proceedings, Zagreb, Croatia, 24-25 September 2008. Croatian Academy of Sciences and Arts, Zagreb, Croatia, pp 75-101

52. RAUŠ $Đ 1996$ Nizinske šume pokupskog bazena. Šumarski institut Jastrebarsko, Radovi 31 (1-2): 17-37

53. RAUŠ Đ, ŠEGULJA N, TOPIĆ J 1985 Vegetacija sjeveroistočne Hrvatske. Glas šum pokuse 23: 225-355

54. GLAVAČ V 1968 Über Eichen-Hainbuchenwälder Kroatiens. Feddes Rep 79 (1-2): 115-138

55. CESTAR D, HREN V, KOVAČEVIĆ Z, MARTINOVIĆ J, PELCER Z 1983 Tipovi nizinskih šuma zapadne Posavine. Šumarski institut Jastrebarsko, Radovi 54: 1-111

56. GLAVAČ V 1961 O vlažnom tipu hrasta lužnjaka i običnoga graba. Sumar List 85 (9-10): 342-347

57. HORVAT I 1938 Biljnosociološka istraživanja šuma u Hrvatskoj. Glas Šum Pokuse 6: 127-279

58. GLAVAČ V 1969 Über die Stieleichen-Auenwälder der SavaNiederung. Schriftner. f. Vegetationskunde, Bad Godesberg 4: 103-108

59. DAKSKOBLER | 2016 Phytosociological analysis of riverine forests in the Vipava and Reka Valleys (southwestern Slovenia). Folia Biologica et Geobotanica 57 (1): 5-61. DOI: https://doi.org/10.3986/fbq0001

60. KOŠIR P, ČARNI A, MARINŠEK A, ŠILC U 2013 Floodplain forest communities along the Mura River (NE Slovenia). Acta Bot Croat 72 (1): 71-95. DOI: https://doi.org/10.2478/v10184012-0015-7

\section{APPENDIX}

Appendix 1.

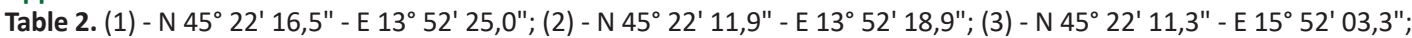

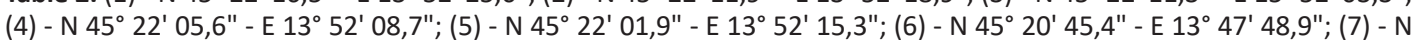

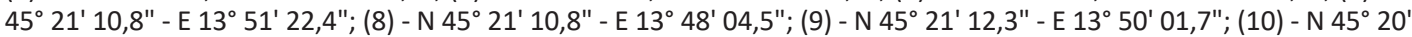
$55,9^{\prime \prime}-\mathrm{E} 13^{\circ} 49^{\prime} 50,4^{\prime \prime}$. 


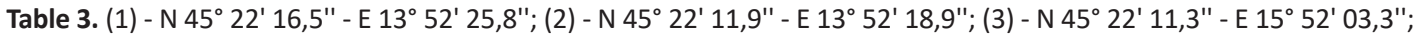

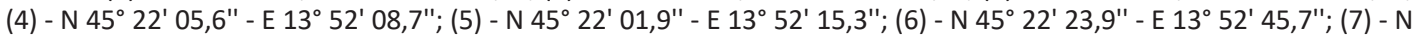

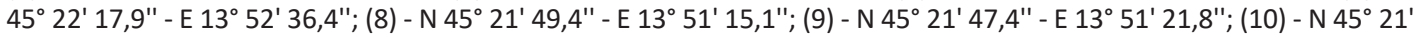

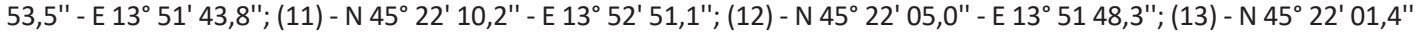

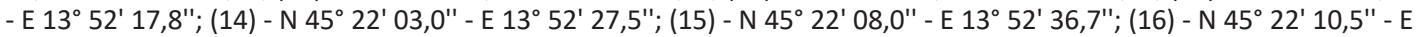

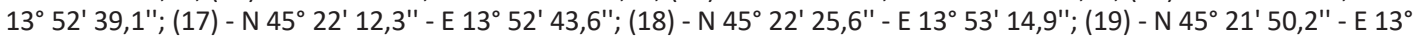

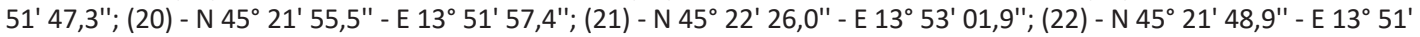
$47,5^{\prime \prime} ;(23)-\mathrm{N} 45^{\circ} 22^{\prime} 36,8^{\prime \prime}-\mathrm{E} 13^{\circ} 53^{\prime} 11,7^{\prime \prime}$.

\section{Appendix 2.}

Alnion incanae Pawlowski in Pawlowski et al. 1928; Alno glutinosae-Fraxinetum angustifoliae Br.-BI. ex Tchou 1948; AlnoQuercion roboris I. Horvat 1938; Alno-Ulmion Br.-BI. et Tüxen ex Tchou 1948; Aro italici-Ulmetum minoris Rivas-Martínez in G. López 1976; Asparago tenuifolii-Quercetum roboris (Lausi 1967) Marinček 1994; Carici pendulae-Quercetum roboris Trinajstić 2008; Carici remotae-Fraxinetum oxycarpae Pedrotti 1970 corr. Pedrotti 1992; Carici remotae-Fraxinion oxycarpae Pedrotti ex Pedrotti et al. in Biondi et al. 2014; Carici remotae-Fraxinetum oxycarpae alnetosum glutinosae Gelini et al. 1986; Carici remotae-Fraxinetum oxycarpae caricetosum cuprinae Mercadal et Vilar 2013; Carici remotaeFraxinetum oxycarpae iridetosum foetidissimae Conti et Pirone 1992; Carici remotae-Fraxinetum oxycarpae populetosum albae Mercadal et Vilar 2013; Carici remotae-Fraxinetum oxycarpae quercetosum pubescentis Mercadal et Vilar 2013; Carici remotae-Fraxinetum oxycarpae quercetosum roboris Mercadal et Vilar 2013; Carici remotae-Fraxinetum oxycarpae crataegetosum laevigatae subass. nova hoc loco; Carpinion betuli Issler 1926; Carpino betuli-Quercetum roboris (Anić 1959) Rauš 1971; Carpino betuli-Quercetum roboris typicum Rauš 1975; Circaeo lutetianae-Quercetum roboris Borhidi 2003; Corno sanguineae-Fraxinetum angustifoliae Lara et Garilleti 1996; Erythronio-Carpinion betuli Marinček in Wallnöfer et al. 1993; Fagetalia Pawlowski in Pawlowski et al. 1928; Ficario ranunculoidis-Fraxinetum angustifoliae Rivas-Martínez et Costa in Rivas-Martínez et al. 1980; Ficario vernae-Ulmetum campestris Knapp ex Medwecka-Kornaś 1952; FraxinoQuercetum roboris Gellini et al. 1986; Fraxino-Ulmetum laevis Slavnić 1952; Fraxino-Ulmetum effusae quercetosum roboris Košir et al. 2013; Fraxino pannonicae-Carpinion betuli Accetto 2006; Fraxino pannonicae-Ulmetum glabrae Aszód 1935 corr. Soó 1963; Genisto elatae-Quercetum roboris I. Horvat 1938; Genisto-Quercetum roboris caricetosum brizoidis I. Horvat 1938; Genisto-Quercetum roboris caricetosum remotae I. Horvat 1938; Genisto-Quercetum roboris carpinetosum betuli Glavač 1961; Junco-Fraxinetum oxycarpae Kárpáti et Kárpáti 1961; Lauro nobilis-Fraxinion angustifoliae Kárpáti et Kárpáti 1961; Leucojo-Fraxinetum angustifoliae Glavač 1959; Leucojo-Fraxinetum angustifoliae typicum Glavač 1959; Leucojo-Fraxinetum angustifoliae alnetosum glutinosae Glavač 1959; Lithospermo purpureocaerulei-Ulmetum minoris Bolòs 1956; Lonicero caprifoliae-Carpinenion betuli Vukelić in Marinček 1994; Lonicero caprifoliae-Quercetum roboris (Rauš) Marinček 1994; Populetalia albae Br.-Bl. ex Tchou 1949; Populion albae Braun-Blanquet ex Tchou 1948; Pseudostellario-Carpinetum betuli leucojetosum aestivi Dakskobler 2016; Pseudostellario-Quercetum roboris Accetto 1974; Querco roboris-Carpinetum betuli (Soó ex Balacs 1943) I. Horvat et al. 1974; Querco roboris-Carpinetum Soó et Pócs 1957; Querco roboris-Carpinetum betuli „submediterraneum“ Bertović 1975; Querco pubescenti-Carpinetum orientalis Horvatić 1939; Querco roboris-Fagetea sylvaticae Br.-Bl. et Vlieger in Vlieger 1937; Rusco-Fraxinetum angustifoliae Gesti et al. 2003; Viburno lantane-Ulmetum minoris Biurrun et García-Mijangos in Rivas-Martínez et al. 2002. 